\title{
A Theory of the Origin of the Solar System
}

\author{
V. MitkA (*) \\ Ricevuto il 18 Agosto 1969
}

\begin{abstract}
SUmmaRy. - A theory of tlie origin of the solar system is propounded. The approach belongs to the dualistic class of theories but still retains all the essential features of a monistic theory. It emphasizes the need of a foreign body approaching the solar nebula for the occurrence of an instability in the boundary layer of the nebular disc. This foreign body is postulated to be a brother star of the Sun in the sense that both belong to a common central parent body such as any two successive planets belong to the Sun. The analysis gives a law of distance of the planets from the Sun. This law is obeyed separately by both the groups of the planets more closely than the existing Titius - Bode's law. The law is also found to be applicable in the various satellite systems.
\end{abstract}

RIAssunto. - Viene proposta. una teoria sull'origine del sistema solare, la quale pur appartenendo alla teoria dualistica, mantiene ancora tutte le caratteristiche essenziali della teoria unitaria. Essa sottolinea la necessità dell'esistenza di un corpo estraneo che avvicina la nebulosa solare nell'eventualità di una instabilità gravitazionale nella fascia limite del disco nebuloso. Si suppone che questo corpo estraneo sia una stella della stessa famiglia del Sole ruotante intorno ad un centro comune, come due pianeti successivi ruotano intorno al Sole. Si perviene poi ad una legge che fornisce le distanze dei pianeti dal Sole. Entrambi i gruppi di pianeti obbediscono a questa legge più rigorosamente di quanto non obbediscano alla legge di Titius Bode. L'A. trova, infine, che la stessa legge può essere estesa a diversi sistemi di satelliti.

\section{INTRODUCTION.}

There have been essentially two different ways of approaching the problem of the origin of the solar system. In the first class of India.

(*) Physics Department, M.N.R . Engineering College. Allahabad, 
thoughts referred as monistic theories, a closed system containing the Sun in the centre and an envelope of rarified gases surrounding it is initially considered. This subsequently, as a result of instability due to the gravitational contraction, splits into a number of rotating gaseous rings. In the second class of thoughts known as dualistic theories, the interaction between the protosun and some foreign body is believed to be the cause of the formation of the planetary system.

The monistic theories initiated by Kant $\left({ }^{25}\right)$ and Laplace $\left({ }^{27}\right)$ have been developed by a large number of workers in various ways by assigning different processes that are responsible for the plitting of the planetary material. The possibility of the occurrence of the gravitational instability due to turbulence was worked out by Weizsâcker $\left({ }^{37}\right)$, Kuiper $\left({ }^{28}\right)$ and Ter Haar $\left({ }^{36}\right)$ and was infact a revival of the very old idea of Descartes $\left({ }^{17}\right)$ vortex theory. The idea of the magnetic field first pointed out by Birkeland $\left({ }^{14}\right)$ was later on used by Alfven $(1,2,3,4)$ to explain the formation of the planetary system. Berlage $\left({ }^{10},{ }^{11},{ }^{12}\right)$, revived the old idea of Kant and studied the possibility of spontaneous splitting of a gaseous volume. Schmidt $\left({ }^{34}\right)$ and his coworkers tried to remove some of the difficulties involved in the various processes of the monistic theories.

The tidal theory first put forward by Buffon $\left({ }^{15}\right)$ was revived by Bickerton $\left({ }^{19}\right)$, Arrhenius $\left({ }^{0}\right)$, Chamberlain $\left({ }^{18}\right)$, Moulton $\left({ }^{32}\right)$, Jeffreys $\left({ }^{23}\right)$ and Jeans $\left.{ }^{(22}\right)$. In fact all these theories are modified forms of Buffon's theory and assume that due to the passage of a star close to the Sun a filament of the form of a cigar with a maximum density in the middle, was drawn out from the surface of the Sun and was consequently broken into the planets.

Binary stars and triple stars theories put forth by Russel $\left({ }^{33}\right)$ and Lyttleton $\left({ }^{28},{ }^{29}\right.$ ) consider the encounter between their component stars. Following Lyttleton's idea, Hoyle $\left({ }^{20}\right)$ investigated the case where a binary companion of the Sun became a supernova. The theory of Whipple ${ }^{(38}$ ) assumes the capture of one small smoke cloud by another big smoke cloud and belongs to the dualistic theories.

The difficulties in the old tidal theories can be summarized as follows:

1. - Rotation of all the planets in the same direction is difficult to conceive.

2. - Small eccentricity of the orbits of all the planets is difficult to understand. 
3. - According to Russell if encounter between a foreign body and the solar nebula is responsible for the formation of the planetary system, one should expect small outer and big inner planets which is contrary to the observations.

4. - Spitzer ${ }^{35}$ ) has shown that the filament drawn out from the surface of the Sun would continuously expand and would not break unless drawn out very fast.

The existence of satellite systems, which are strikingly similar to the planetary system, cannot be ignored while propounding any theory of the origin of the solar system (Alfven ( $\left.{ }^{\circ}\right)$ ). Any acceptable theory should be a general one and should explain the formation of any system of secondary bodies around a parent body.

In the present paper an attempt has been made to formulate a theory which can explain the formation of planetary system as well as satellites systems. The approach essentially belongs to the dualistic side but still retains all the salient features of the monistic theory. In fact it takes essence of both the theories and combines them in such a wey that all the above difficulties of the old tidal theories are removed.

The present theory envisages the need of a foreign body approaching the solar nebula for the development of an instability and hence for the splitting of the gaseous rings from the boundary of the solar nebula. This body is postulated to be a brother star of the Sun in the sense that both the Sun and the brother star were revolving at the time of their formation round a common central parent body just as any two successive planets revolve round the sun.

The present analysis gives a law of distance of the planets from the Sun which is obeyed separately by the outer and the inner groups of the planets more closely than the existing Titius-Bode's law. In fact the law of distance so obtained is not only obeyed by the family of the Sun, but also by the various families of the planets.

\section{ORIGIN OF THE SOLAR NEBULA.}

In the absence of any real knowledge about the birth of the solar nebula, only speculations can be made which can stand all the way to explain the salient features of the solar system and its related facts. While making such speculations, one should look upon the family of the Sun as well as the families of the planets for guidance. An 
inspection of the families of the planets reveals that they show the same salient features as exhibited by the family of the Sun - the coplaner orbits of small eccentricity. This indicates that all might have been formed through the same stages of evolution. The idea may also be extended to one generation back, that is, the Sun along with its few brother stars might have been formed by the same course of evolution and at some stage might be revolving in coplaner and nearly circular orbits around their parent body just as the planets move round the Sun and the satellites round the planets. If this is true, then the solar nebula must have been formed as a result of the condensation of a gaseous ring.

Since most of the celestial bodies exhibit isochronism in their axial rotation; it suggests that the formation of a body is not a consequence of direct condensation of a gaseous cloud (Alfven ( ${ }^{7}$ )). A two step agglomeration has been suggested. First the gaseous cloud condenses into a number of small grains and these are later accreted by the gravitational attraction of a growing embryo. Formation of the planetary bodies by particle agglomeration has been described in different ways by Schmidt $\left({ }^{34}\right)$, Alfven $\left({ }^{5}\right)$, Marcus $\left({ }^{30}\right)$ and Giuli $\left({ }^{10}\right)$. Hence irrespective of the process how grains and an embryo are formed in a rotating gaseous ring, it may be assumed that once they are formed the embryo will go on gathering material as it moves in its orbit.

The grains produced in the rotating gaseous rings move in their respective Keplerian orbits prior to their accretion by the moving embryo. As a result of impacts of these grains on the embryo, the latter acquires spin angular momentum. This picture of particle agglomeration is exactly similar to that described by Giuli $\left({ }^{19}\right)$. The torque, which any colliding particle exerts on the condensing nucleus due to such impacts, should increase as the size of the forming body increases. Therefore most of the spin angular momentum shall be built up in the later stages of the condensation of the dust cloud, that is, when the rarified envelope is under formation. By this time the major portion of the mass is already collected and condensed to form a heavy and small core at the centre. Therefore the spin angular momentum is mostly confined to the envelope. Later on, due to the gravitational contraction of the envelope a rotating disc is formed in the equatorial plane of the forming body.

It may be pointed out that the material of the nebular disc so formed must be nearly in gaseous form. The accreted particles after 
falling from long distances must have attained high velocities before colliding and absorbed in the envelope of the forming body. Such a collision should result not only in vaporization but ionisation also to some extent. In the initial stages, therefore, the envelope might contain some plasma as decribed by Alfven $\left(^{8}\right)$ in his theory of partial corotation of plasma. Action of the dipole moment of the central body over the plasma in the envelope might have also played some role in establishing the angular momentum of the envelope. It is, however, assumed in the present study that all such actions were over due to condensation of plasma into neutral gases before the formation of the planetary system.

The solar nebula and the nebula of its brother star, both moving in adjacent orbits, are assumed here to be formed simultaneously and in a similar way. These two move in their respective orbits with different but constant angular velocities and hence the distance between their centres changes continuously with time. The gravitational contraction in the gaseous disc of the solar nebula will now progress under a continuously changing tidal force due to the brother star. As the size of the nebular disc of the Sun decreases due to the gravitational contraction, a stage may be reached when an instability arises in its boundary layer. $\Lambda$ t this stage the gravitational force on unit mass of the boundary layer becomes comparable to its centrifugal force. Prior to this stage the gravitational contraction of the solar nebula proceeds without any appreciable viscous loss and the solar nebula spins more or less like a rigid body. When the instability arises in the boundary layer, it will tend to develop angular velocity gradient among its different layers. But this is continuously apposed by a viscous loss and therefore with the start of the instability the viscous loss increases. On account of this continuous loss of angular momentum the boundary layer is not able to attain exact Keplerian velocity. As such the nebula and its disc contract continuously by slowly losing energy and angular momentum without separation of any gaseous ring at the boundary.

If the viscous loss in the boundary layer is overcome by a continuous supply of energy from some external agency, it will then attain an exact Keplerian velocity. After the attainment of exact Keplerian velocity this boundary layer will rotate in a fixed orbit while all the inner layers which have to attain Keplerian velocity, will contract further till finally the boundary layer splits from the main body in the form of a rotating gaseous ring. It is assumed here that energ: 
required to overcome this viscous loss in the boundary layer is provided by the brother star of the Sun during that part of its orbital motion when it is approaching towards the solar nebula.

\section{THEORY.}

At any instant the distance $R$ between the solar nebula and its brother star is given by

$$
R^{2}=R_{1}^{2}+R_{2}^{2}+2 R_{1} R_{2} \cos \phi
$$

where $R_{1}$ and $R_{2}$ are respectively the orbital radii of the solar nebula and the star and $\phi=\pi-\psi, \psi$ being the angle between the radius vectors. The angle $\phi$ increases as the solar nebula and the star approach each other. If the Sun and the star move in their orbits with angular velocities $\omega_{1}$ and $\omega_{2}$ respectively, the rate of change of $\phi$ with time can be written as

$$
\frac{d \phi}{d t}=\omega_{1}-\omega_{2}
$$

The distance $R$ between the Sun and the star changes continuously. As a result the combined potential energy of the Sun and its brother star should change. On order to conserve the total energy of the combination, their kinetic energies change accordingly. For example, if during any part of the orbital motion the star approaches nearer to the Sun, then $R$ decreases which results in an increase of the kinetic energy of any mass situated on either of them. The gravitational potential energy $V$ of the combination of the solar nebula and its brother star, when they are situated $R$ distance apart, is given by

$$
v=-\frac{G}{R} \frac{M}{M^{\prime}}
$$

where $M$ and $M^{\prime}$ are respectively the masses of the solar nebula and its brother star and $G$ is the gravitational constant. The decrease in this energy per unit time due to the decrease in $R$ is

$$
\frac{d V}{d t}=\frac{G M M^{\prime}}{R^{2}} \frac{d R}{d t} .
$$

This energy will appear as an additional kinetic energy of the system one second. This kinetic energy will be shared by the solar 
nebula and the star in the ratio of their masses. The share received by the solar nebula will be

$$
\frac{M}{\left(M-M^{\prime}\right)} \frac{G M M^{\prime}}{R^{2}} \frac{d R}{d t} .
$$

The increase in the kinetic energy per second per unit mass in the solar nebula is

$$
\frac{G M M^{\prime}}{\left(M+M^{\prime}\right) R^{2}} \frac{d R}{d t} .
$$

Any unit mass in the boundary layer of the solar nebula has angular motion about the parent star as well as about the axis of the Sun. Therefore out of the above increase in the kinetic energy only a certain portion will enhance the motion of the unit mass about the Sun, while the rest will enhance its motion about the parent star. The division of this energy between the two motions can be made by assuming that the two shares of the energy shall produce equal instability in their respective motions. By equating the resulting additional centrifugal force in the two motions, it can be shown that the energy is shared in the ratio of the orbital radii of the two motions. The part $E$ of the kinetic energy received per second by the unit mass on the surface of the solar nebula, which enhances its motion about the Sun, is given by

$$
E=\frac{G M M^{\prime} r}{\left(M+M^{\prime}\right)\left(R_{1}+r\right) R^{2}} \frac{d R}{d t} .
$$

Since the radius $r$ of the orbit of the assumed unit mass about the Sun is very small as compared to the radius $R_{1}$ of its orbit about the parent star, expression [6a] reduces to

$$
E=\frac{G M M^{\prime} r}{\left(M+M^{\prime}\right) R_{1} R^{2}} \frac{d R}{d t} .
$$

It may be pointed out that due to mutual attraction, a simultaneous gain of kinetic energy in the orbital motions of the solar nebula and its brother star is not expected. In fact if there is a gain of kinetic energy in the orbital motion of one, there will be loss in the other because these motions are governed by the gravitational force of a common central body. However, there is no such binding in their spin motions and therefore these motions will have a gain of kinetic energy as it would be when the two bodies would have independent orbital motions. Therefore in the above treatment for obtaining the 
gain of kinetic energy in the spin motions, the Sun and the brother star have been assumed to be moving in independent orbits. Moreover the above method of the distribution of the gained kinetic energy between the two angular motions of a unit mass situated in the solar nebula, applies only when the mass situated in the boundary layer is nearing Keplerian velocity. The inner layers, being quite far from the stage of Keplerian velocity, continuously suffer gravitational contraction inspite of continuous gain of some angular momentum and energy due to the approaching star. The gain of kinetic energy by a unit mass of the solar nebula per second will be very small so that any layer takes quite a long time to attain Keplerian velocity.

For calculating the viscous loss per unit mass per second in the boundary layer of the nebular dise at a stage when it is nearing Keplerian velocity, we consider a thin cylindrical layer of radius $r$ and thickness $\delta r$ in the boundary. The net viscous drag $\mu$ experienced by such a layer is given by

$$
\mu=\frac{d}{d t}\left(2 \pi r T \eta r \frac{d \omega}{d r}\right) \delta r,
$$

where $\eta$ is the coefficient of viscosity of the gas in the considered layer, $T$ is the height of the layer and $\omega$ is its angular velocity. Substituting the value of $\omega$ and noting that $\eta$ varies directly as the square root of the absolute temperature 0 , Eq. [7a] becomes

$$
\mu-\frac{d}{d r}\left(-3 \pi \eta_{o} T r^{2} \frac{\sqrt{\theta}}{\sqrt{\bar{\theta}_{o}}} \frac{\sqrt{G M}}{r^{5 / 2}}\right) \delta r
$$

where $\eta_{n}$ is the coefficient of viscosity of the gas in the boundary of the gaseous ball of radius $r_{o}$ and temperature $0_{o}$. Assuming that the absolute temperature of a layer of the nebular disc varies inversely with some power $n^{\prime}$ of the radius, we have

$$
\mu=\frac{3}{2}\left(n^{\prime}+1\right) \frac{\pi \eta_{0} \frac{T}{T} r_{o}^{n^{\prime} / 2} \sqrt{G M}}{r^{\left(n^{\prime}+3\right) / 2}} \delta r .
$$

The viscous drag $\mu^{\prime}$ experienced by the unit mass is given by

$$
\mu-\frac{3}{4}\left(n^{\prime}+1\right) \frac{n=\sqrt{G M} r_{o}^{n^{\prime} / 2}}{\varrho r^{\left(n^{\prime}+5\right) / 2}} .
$$


The torque $\tau$ experienced by the unit mass due to this viscous drag is

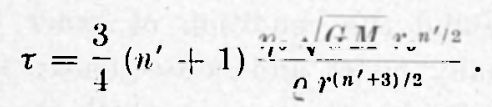

The loss of the energy $E^{\prime}$ per unit mass per second in the bounpary layer of the nebular disc due to the viscous drag is given by

$$
\begin{aligned}
E^{\prime} & =\frac{3}{4}\left(n^{\prime}+1\right) \frac{\eta_{0} \sqrt{(\dot{H} M} r_{o^{n^{\prime}} / 2} \omega}{\varrho r^{\left(n^{\prime}+3\right) / 2}}, \\
& =\frac{3}{4}\left(n^{\prime}+1\right) \frac{\eta_{0} G M r_{o^{n^{\prime} / 2}}}{\varrho r^{n^{\prime}+3}} .
\end{aligned}
$$

Equating the kinetic energy [6b] to the viscons loss [10] we obtain $r^{\frac{n^{\prime}}{2}+4}=\frac{3}{4}\left(\eta^{\prime}+1\right) \frac{\left(M+M^{\prime}\right) n_{0} R_{1} r_{o}^{n^{\prime} / 2}\left(R_{1}{ }^{2}+R_{2}{ }^{2}+2 R_{1} R_{2} \cos \phi\right)^{3 / 2}}{\left(\omega_{1}-\omega_{2}\right) M^{\prime} R_{1} R_{2} \varrho \sin \phi}$

Now we assume that the density $\varrho$ in any layer of the nebular disc varies inversely as the $n^{t h}$ power of the radius of the layer so that

$$
\varrho=\frac{K}{r^{n}},
$$

where $K$ is a constant. Settino

$$
\left[\frac{3}{2}\left(n^{\prime}+1 ; \frac{\left(M+M^{\prime}\right) \eta_{0} r_{0}^{n^{\prime} / 2}\left(R_{1}^{2}+R_{2}^{2}\right)^{3,2}}{2 K\left(\omega_{1}-\omega_{2}\right) M^{\prime} R_{1} R_{2}}\right]^{\frac{1}{n^{\prime}}-n+4}=\Lambda\right.
$$

and

$$
\frac{2 R_{1} R_{2}}{R_{1}^{2}+R_{2}^{2}}=B
$$

the relation [11] can be written as

$$
r=\frac{A(1+B \cos \phi)^{3 / n^{\prime}-2 n+8}}{(\sin \phi))^{\frac{1}{n^{\prime}}-n+4}} .
$$

The angle $\phi$ in Eq. [14] varies with time and therefore this equation gives the position of that layer in the nebular disc where exact Keplerian velocity is attained at time $t$. As the brother star of the Sun comes nearer and nearer to the solar nebula, the position of exact Keplerian velocity shifts towards inner layers. However this shift of the condition of exact Keplerian velocity is slow due to the small 
value of $\frac{d \phi}{d t}$. On the other hand inner layers of the nebular disc which are much behind the condition of exact Keplerian velocity, contract gravitationally faster and consequently the boundary layer may get separated. During the time in which the splitting of a boundary layer progresses, the remaining body contract gravitationally. When the splitting of a gaseous ring is matured, the boundary layer in the remaining nebula is again nearing Keplerian velocity. At this moment condition [14] may again be satisfied at some layer of the remaining nebula. Thus at the time when the splitting of a boundary layer is just complete, the condition of exact Keplerian velocity jumps from the split layer to some other layer in the boundary of the remaining nebula. The remaining nebula undergoes the same process again and finally meets the same fate, so that again a gaseous ring is split. In this way in the total span of $180^{\circ}$ for $\phi$, during which the brother star approaches the solar nebula, Eq. [14] is satisfied in the nebular disc at a number of places and each time producing one gaseous ring. If $\varrho$ in $\mathrm{Eq}$. [14] be taken to represent the average density in any such split ring, this equation will give the position of the various gaseous rings provided we know the value of $\phi$ corresponding to each gaseous ring. As the orbital radius of any of these rings will not change even if it is condensed to form a planet, Eq. [14] will give the law of distance of the planets from the Sun.

In order to know the value of $\phi$ corresponding to each split gaseous ring it is required to study how density of the gas varies in any given layer of the nebular disc. In a layer of radius $r$, the total radial force $F^{\prime}$ acting on a gaseous element of length $\delta r$ and unit cross-section is given by

$$
F=\frac{d(\varrho v)}{d t} \delta r=\frac{G M \varrho}{r^{2}} \delta r-r \omega^{2} \varrho \delta r+\delta P
$$

where $\delta P$ is the difference of pressure on the two faces of the gaseous element. Also for the same layer the equation of continuity is

$$
\frac{d(\varrho v)}{d r}=\frac{d \varrho}{d t}
$$

where $v$ is the velocity of the inward flow at a radius $r$. If 0 be the 
absolute temperature of the gas in the layer of radius $r$ then, the pressure $P$ is given by

$$
P=\frac{\bar{R} \theta \varrho}{\bar{M}}
$$

where $\bar{R}$ is the gas constant and $M$ is the molecular weight. Substituting the value of $\delta P$ in Eq. [15] we get

$$
\frac{d(o v)}{d t}=\frac{\bar{R} \theta}{M} \frac{d \rho}{d r}+\frac{G M}{r^{2}}-r \omega^{2} \rho .
$$

From Eqs. [16] and [18] we get

$$
\frac{d^{2} \varrho}{d t^{2}}=\frac{\bar{R} \theta}{M} \frac{d^{2} \varrho}{d r^{2}}, \frac{d}{d r}\left[\left(\frac{G M}{r^{2}}-r \omega^{*}\right) \varrho\right] .
$$

In Eq. [19] $\omega$ is a function of $r$ ant $t$. It will be assumed in the present study that $\omega$ varies with time in such a way that $\varrho$ is a symmetrical function of $t$. During gravitational contraction, the density in any layer of the nebular disc is expected to exhibit a maximum at a certain time and about this time the variation of density will be symmetrical. It means that the time, in which the maximum density is accomplished at a certain layer, will be equal to the time in which the density will vanish.

It si assumed that initially the solar nebula extended to a large size and in the region of nebular disc there existed everywhere a very much rarified density. This rarified density continued to be there till for the first time Eq. [14] became applicable. Upto this moment the density in the nebular disc did not change appreciably by gravitational contraction and the density at any place in the disc was negligibly low as compared to the maximum density to be attained at certain later stage. Counting this moment to be zero time, the time in which maximum density is attained in any layer should be equal to the time in which the density is reduced to zero. The reduction of density to zero at a certain layer marks that moment when a gaseous ring in the boundary region splits and at the same time condition [14] jumps from the split layer to the next. Upto this moment the place at which the next gaseous ring will split, has been gaining density and just from this moment onward it will be losing. Consequently the time in which a certain gaseous ring sphts is double of the time in which its preceding gaseous ring is split. Thus if $s$ refers to the 
order of a gaseous ring starting from the outer most, the angle $\phi$ corresponding to it is given by

$$
\phi=2^{s-1} 0+\theta_{o},
$$

where 0 is the value of $\phi$ between the first and the second gaseous rings and $0_{o}$ is the initial value of $\phi$ when the condition [14] became applicable for the first time. Substituting this value of $\phi$ in Eq. [14] we get the desired law of distance of the planets from the Sun as

$$
r=\frac{A\left[1+B \cos \left(2^{s-1} 0+\theta_{o}\right)\right]^{3 / n^{\prime}-2 n+8}}{1} .
$$

The above law is general and is applicable to any sequence of bodies formed from the parent body in the way considered above. The explicit form of it, applicable to any group of planets or sequence of satellites, may be obtained by putting the value of $\left(\frac{n^{\prime}}{2}-n\right)$ which depends upon the density and temperature distributions in the original gaseous volume producing the system.

\section{Application to Planfarary System.}

Eq. [21] contains a number of parameters namely, $A, B, 0,0_{0}$ and $\left(\frac{n^{\prime}}{2}-n\right)$. The values of some of them can easily be lixed. For example, the value of 0 can be obtained from the knowledge of the number of planets which were formed in one sequence in a total span of $180^{\circ}$ for $\phi$. Following our earlier investigation $\left({ }^{31}\right)$, that all the planets were not formed in one succession but in two groups, the relation [21] should be applied to both the groups of the planets separately. In the outer group, five planets were formed roughly in a total span of $\phi=180^{\circ}$. Therefore putting $2^{s-1} \theta=180$ and $s=5,0$ is found to be $11.25^{\circ}$. For $s=6,0$ is $5.6^{\circ}$. As five planets were completely formed and the sixth one could not be completed, the limits of $\theta$ for the outer group are: $5.6^{\circ}<0<11.25^{\circ}$. As an approximation the mean value of the above two limits may be taken as the value of $\theta$ for the outer group of the planets. Thus for the outer group 0 is $8.5^{\circ}$ approximately. Similarly for the inner group of the planets in which four planets were formed in one succession and fifth could not be completed, $\theta=17^{\circ}$ approximately. 
Regarding the value of $\theta_{o}$, it may be pointed out that if a sequence of planets is formed after when already few planets of some preceding sequence have been formed, then the value of $\theta_{o}$ will be zero. This is so because after the formation of the previous sequence the nebula and the star would be receding from each other for a span of $\phi=180^{\circ}$. Hence the solar nebula gets enough time to establish instability in its boundary layer before Eq. [21] becomes applicable at $\phi=0$ from where it starts approaching the brother star. Thus $\theta_{o}$ should be equal to zero for the inmer group of the planets but may have some finite value for the outer group of the planets.

All approximate idea of the value of the constante $B$ can be had from the expression [13b]. In this expression $R_{1}$ and $R$, are respectively the orbital radii of the solar nebula and its brother star. An inspection of the ratio of the orbital radii of any two successive bodies in any group of planets or satellites reveals that the ratio $R_{2} / R_{1}$ increases with the size of the central body. The value of $R_{2} / R_{1}$ is roughly 2 for the family of the Sun and that of Jupiter. A still higher value of $R_{2} / R_{1}$ should rather suit for the family of the parent star of the Sun. Taking tentatively $R_{2} / R_{1}=2.5$, the value of $B=2 / 3$ is obtained.

The best-fit values of the various parameters for the two groups of the planets are as follows:

OUTER Group:

$$
\begin{aligned}
A & =8.913 \text { a.u. } \\
B & =\frac{2}{3} \\
\theta & =8.5^{\circ} \\
\theta_{o} & =18.5^{\circ} \\
\left(n-\frac{n^{\prime}}{2}\right) & =3 .
\end{aligned}
$$

INNER GROUP:

$$
\begin{aligned}
A & =0.5690 \text { a.u.. } \\
B & =\frac{2}{3} \\
\theta & =17^{\circ} \\
\theta_{0} & =0^{\circ} \\
\left(n-\frac{n^{\prime}}{2}\right) & =2 .
\end{aligned}
$$


The values of the orbital radii of all the planets in each group have been determined by using the above parameters. The calculated values are given in Tables I and II together with the observed values which agree fairly well.

Table I - COMPaRISON OF THE OBSERVED AND TIIE CALCULATED ORBITAL RADII OF THE PLANETS OF THE OUTER GROUP.

\begin{tabular}{|l|c|c|}
\hline Planets & $\begin{array}{c}\text { Calculated } \\
\text { orbital radii }\end{array}$ & $\begin{array}{c}\text { Observed } \\
\text { orbital radii }\end{array}$ \\
\hline Pluto & 39.52 & 39.52 \\
Neptune & 29.42 & 30.07 \\
Uranus & 18.73 & 19.19 \\
Saturn & 9.479 & 9.539 \\
Jupiter & 5.203 & 5.203 \\
\hline
\end{tabular}

Table II - Comparison of the observed and the CaLCULATEd orbital RADII OF THE PLANETS OF THE INNER GROUP.

\begin{tabular}{|l|c|c|}
\hline Planets & $\begin{array}{c}\text { Calculated } \\
\text { orbital radii }\end{array}$ & $\begin{array}{c}\text { Observed } \\
\text { orbital radii }\end{array}$ \\
\cline { 2 - 3 } Mars & 1.523 & 1.523 \\
Earth & 1.058 & 1.000 \\
Venus & .698 & .723 \\
Mercury & .482 & .400 \\
\hline
\end{tabular}

V. APPLICATION TO SATELLITE SYSTEMS.

Equation [21], which has been derived for a contracting nebula under the continued tidal force of an approaching brother nebula, is 
quite general and can be applied to any system of bodies so formed. The sequences of the satellites of the big planets can be assumed to have been formed in a similar way and should satisfy Eq. [21]. The parameters of Eq. [21] for the different satellite systems can be determined more definitely because their parents bodies (Planets) are precisely known. Three such sequences each consisting of five satellites and belonging to Jupiter, Saturn and Uranus respectively can be easily picked up from the satellite data. Jupiter and Saturn are known to contain a number of satellites other than the considered group of five satellites. These remaining satellites either may be belonging to some incomplete groups formed in a similar way or may be captured bodies of their parent planets. The three satellite systems shall be studied here:

Satellites of Jupiter. An inspection of the value of $R_{2} / R_{1}$ shows that the five inner satellites of Jupiter form a sequence like the outer group of the solar system. The value of 0 for this sequence of satelhtes should, therefore, be taken as 8.5०. The value of $\theta_{o}$ will be zero because a number of satellites exist beyond this sequence. Saturn, being the heaviest and closest planet to Jupiter, may be considered solely responsible for exerting necessary tidal force on the surface of Jupiter for the formation of its satellites. From the values of the orbital radii of Jupiter and Saturn, the numerical value of $B$ comes out to be 0.84 . In this way, only two parameters $A$ and $\left(n-\frac{n}{5}\right)$ remain to be fixed in Eq. [21]. The values of the different parameters of Eq. [21] as applied to the satellites of Jupiter are:

$$
A=4.025 a_{8}, \quad B=0.84, \quad \theta=8.5^{\circ}, \quad \theta_{o}=0^{\circ}
$$

and

$$
\left(n-\frac{n^{\prime}}{2}\right)=\frac{5}{2},
$$

where $a_{s}$ is the radius of Jupiter. The orbital radii of the various satellites of Jupiter as calculated with the help of Eq. [21] are compared with their observed values in Table III.

Satellites of Saturn: The inner five satellites of Saturn also form a sequence similar to that of Jupiter. The heaviest and closest planet which can have the maximum tidal influence upon the surface of Saturn is Jupiter. From the orbital radii of Jupiter and Saturn, the value of the constant $B$ is $\mathbf{0 . 8 4}$. Following the previous argument, $\theta=8.5^{\circ}$ 
and $\theta_{0}=0^{\circ}$ for this case also. Again the two remaining parameters $\left(n-\frac{n}{2}\right)$ and $A$ are to be adjusted empirically. Various parameters of $\mathrm{Eq} .[21]$ as applied to the satellites of Saturn are:

$$
A=3.452 a_{s}, \quad B=0.84, \quad \theta=8.5^{\circ}, \quad \theta_{0}=0^{\circ}
$$

and

$$
\left(n-\frac{n^{\prime}}{2}\right)=1
$$

Table III - CoMparison of tile observed aNd tile calculated orbital

\begin{tabular}{|c|c|c|}
\hline Satellites & $\begin{array}{l}\text { Calculated } \\
\text { orbital radii }\end{array}$ & $\begin{array}{l}\text { Observed } \\
\text { orbital radii }\end{array}$ \\
\hline Gallisto (VI) & 26.36 & 26.36 \\
\hline Ganyuade & 16.52 & 14.99 \\
\hline Europe & 10.19 & 9.396 \\
\hline$I\left(I_{0}\right)$ & 5.886 & 5.905 \\
\hline$V$ & 2.547 & 2.539 \\
\hline
\end{tabular}
RADII OF THE SATELLITES OF JUPITER.

Table IV - Comparison of the observed and the Calculated orbitai RADil OF THE SATELlites of SATURN.

\begin{tabular}{|l|c|c|}
\hline Satellites & $\begin{array}{c}\text { Calculated } \\
\text { orbital radii }\end{array}$ & $\begin{array}{c}\text { Observed } \\
\text { orbital radii }\end{array}$ \\
\cline { 2 - 3 } Rhea & 8.835 & 8.835 \\
Dione & 6.979 & 6.327 \\
T'ethys & 5.456 & 4.939 \\
Linceladus & 4.058 & 3.991 \\
Mima & 2.502 & 3.111 \\
\hline
\end{tabular}


where $a_{s}$ is the radius of Saturn. The orbital radii of different satellites of Saturn as calculated from Eq. [21] are given in Table IV together with their observed values.

Satellites of Uranus: The five satellites of Uranus also form a similar sequence. Therefore $\theta=8.5^{\circ}$ and $n_{0}=0^{\circ}$. The closest planet which has the maximum tidal effect on the surface of Uranus is Saturn. From the orbital radii of Uranus and Saturn, the value of the constant $B=0.8$. The remaining two parameters $A$ and $\left(n-\frac{n}{2}\right)$ are adjusted empirically. The different parameters of Eq. [21] as applied to the satellites of Uranus are:

$$
A=9.8 a_{U}, \quad B=0.8, \quad 0=8.5^{\circ}, \quad 0_{0}=.0^{\circ}
$$

and

$$
\left(n-\frac{n^{\prime}}{2}\right)=1.75
$$

where $a_{U}$ is the radius of Uranus. The orbital radii of dillerent satellites of Uranus as calculated with the help of Eq. [21] are compared with their observed values in Table $\mathrm{V}$.

Table V - Comparison of THE OBSERVED aND TIIE CalCulated oRitital RADII OF THE SATELLITES OF URANUS.

\begin{tabular}{|l|c|c|}
\hline Satellites & $\begin{array}{c}\text { Calculated } \\
\text { orbital radii }\end{array}$ & $\begin{array}{c}\text { Observed } \\
\text { orbital radii }\end{array}$ \\
\cline { 2 - 3 } Oberon & 24.69 & 24.69 \\
Titania & 18.05 & 18.46 \\
Umbriel & 13.00 & 11.25 \\
Ariel & 8.814 & 8.079 \\
Miranda & 4.752 & 5.494 \\
\hline
\end{tabular}

VI. Evaludation of the constant A.

For any sistem of satellites or planets the value of the constant $A$ can be evaluated with the aid of the expression [13a]. For the satel- 
lite system, all the quantities except $\eta_{0}, n, n^{\prime}$ and $K$ are known. However, these quantities can also be estimated approximately for each satellite system as follows:

$\eta_{0}:$ In the expression [13a] $\eta_{0}$ refers to the coefficient of viscosity of the gases in the boundary layer of the original gaseous volume of the planet when its radius was $r_{0}$. The radius $r_{0}$ can be taken approximately equal to the orbital radius of the outermost satellite of the planet. At the time when not a single satellite was formed and the whole gaseous volume of the planet was intact, its surface temperature would approximately be equal to the black body temperature in equilibrium with the Sun. Assuming the temperature of the surface of the Sun to be about $6000 \mathrm{o}$, the black body temperatures of the surface of Jupiter, Saturn and Uranus come out to be $120^{\circ} \mathrm{K}, 88^{\circ} \mathrm{K}$ and $620 \mathrm{~K}$ respectively. Remembering that in Jupiter and Saturn there is mostly hydrogen and in Uranus it is metahne, the value of $\eta_{0}$ for all these planets can be approximately known from the experimental values of the viscosity of these gases. The values of $\eta_{0}$ used in the present study for diflerent planets are depicted in Table VI.

Table VI - Values of the constants $\eta_{o}, n, n^{\prime}$ and $K$ for the sateli.ite srstems of Jupiter, Saturn and Dranus.

\begin{tabular}{|l|c|c|c|c|}
\hline Satellite system & $\begin{array}{c}\eta_{0} \\
\text { (micro poise) }\end{array}$ & $n$ & $n^{\prime}$ & $\begin{array}{c}K \\
\text { (C.C.S.) }\end{array}$ \\
\cline { 1 - 2 } Jupiter & 45 & 3.50 & 2 & $3.835 \times 10^{30}$ \\
Saturu & 57 & 2.00 & 2 & $1.180 \times 10^{13}$ \\
Urauus & 38 & 2.75 & 2 & $8.189 \times 10^{20}$ \\
\hline
\end{tabular}

$n$ and $n^{\prime}$ : The value of $\left(n-n_{0}^{n}\right)$ is already known for each satellite system. Assuming the radial distribution of temperature in the original gaseous volume of the planets to be the same as in the solar nebula, the same value of the index $n^{\prime}=: 2$ may be taken for all the planets. In this way the values of $n$ and $n^{\prime}$ may be known separately for any system and these are tabulated in Table VI.

$K$ : In order to calculate the value of the constant $K$, it will be assumed that a satellite is formed at the centre of gravity of the 
radial mass distribution of a gaseous ring. In this way the external and internal radii of any gaseous ring can be shown to be linear function of the orbital radius of the planet formed out of it $\left(^{31}\right)$. This enables us to find the internal and external radii of any gaseous ring. For example in the case of Jupiter, its satellite (I) $\mathrm{I}_{0}$ has mass $(3.81 \pm 0.3) \times 10^{-\mathrm{s}}$ times the mass of Jupiter. The external and internal radii $r^{\prime \prime}$ and $r^{\prime}$ of the gaseous ring which might have given rise to this satellite come out to be $8.487 a_{J}$ and $4.442 a_{J}$ respectively. Thus the equation determining the value of $K$ for Jupiter can be written as

$$
\frac{K}{r_{(1)^{3 \cdot 5}}}=\frac{m_{(\mathrm{I})}}{\pi\left(r^{\prime \prime 2}-r^{\prime 2}\right) T^{\prime}},
$$

where $r_{(\mathrm{I})}$ and $m_{(\mathrm{I})}$ are respectively the orbital radius and mass of the satellite $(\mathrm{I}) \mathrm{I}_{\mathrm{o}}$ and $T$ is the thickness of the gaseous disc. The thickness $T$ of the disc may be taken tentatively equal to the orbital diameter of the innermost satellite of the system. Thus $T=2.539$ times the diameter of Jupiter. Eq. [22] then gives the value of the constant $K$ as $3.835 \times 10^{30}$ C.G.S. for the satellites of Jupiter. The value of the constant $K$ for the other satellite system can be determined in a similar manner and these are given in Table VI.

Table VII - Comparison of the empirical and the evaluated values OF THE CONSTANT $A$ FOR VARIOUS SATELLITE SYSTEMS.

\begin{tabular}{|l|c|c|}
\hline Satellite system & $\begin{array}{c}\text { Evaluated values } \\
\text { (Light years) }\end{array}$ & $\begin{array}{c}\text { Empirical values } \\
\text { (Light years) }\end{array}$ \\
\cline { 1 - 2 } Jupiter & 1.330 & 4.025 \\
Saturn & 7.176 & 3.452 \\
Uranus & 12.53 & 8.098 \\
\hline
\end{tabular}

With the help of the values of the parameters $\eta_{o}, n, n^{\prime}$ and $K$ from Table VI, the values of the constant $A$ have been determined from the expression [13a]. The calculated values of $A$ for various satellite systems are given in Table VII and compared with those obtained empirically by adjusting the parameters of Eq. [21]. A perusal of the Table VII shows a fair agreement between the two sets 
of values. Better agreement is not expected in view of the use of approximate values of some quantitaties. For example the value of viscosity $\eta_{0}$ is uncertain due to lack of the exact knowledge of the composition of the satellite material, and that of $n$ and $n$ ' due to lack of the exact knowledge of the density and temperature distributions in such bodies. This agreement of the values allows us to obtain some information about the parent star of the Sun. In the expression [13a] the quantities which cannot be determined are $M^{\prime}, R_{1}, R_{2}$ and the mass $M_{o}$ of the parent star of the Sun. However, under certain approximations the order of magnitude of $H_{0}$ and $R_{1}$ can be known. Remembering that for the outer group of the planets of the Sun, $n^{\prime}=2$ and $n=4$, expression [13a] can be written as

$$
\Lambda=\frac{9 \eta_{0} r_{o}\left(1+M / M^{\prime}\right) R_{1^{3 \cdot 5}}\left[1-\left(R_{2} / R_{1}\right)^{2}\right]^{1 / 2}}{2 K B \sqrt{G M}\left[1 \sim 1 /\left(R_{2} / R_{1}\right)^{1 \cdot 5}\right.}
$$

In this equation the quantities $R_{2} / R_{1}, M^{\prime} / M, M_{0}$ and $R_{1}$ are unknown. Out of these $M / M^{\prime}$ and $R_{1} / R_{2}$ are the ratios of the masses and that of the orbital radii of the Sun and its brother star respectively. The ratio $R_{2} / R_{1}$ has already been fixed as 2.5 in the determination of the constant $B$ for the planetary system. It is to be noted that the value of the constant $B$ depends upon the ratio of the orbital radii of the Sun and its brother star but it does not ascertain whether $R_{2}$ is 2.5 times $R_{1}$ or vice versa. Thus both the possibilities may be examined. The ratio $M^{\prime} / M$ may be taken equal to be unity because the masses of the Sun and its brother star would be of the same order of magnitude. The remaining two unknown quantities in the expression [23] are $M_{o}$ and $R_{1}$. The parent star of the Sun as postulated in the present study may be identified with a first generation objest described by Doroshkevitch et $\Lambda 1 .\left({ }^{18}\right)$. Now the distance $R_{1}$ can be determined from [23]; other constants e.g. $\eta_{0}, K, r_{0}$ etc. being determined exactly in a similar manner as for the satellite systems.

The second possibility, in which the orbit of the solar nebula is outside the orbit of its brother star, gives the orbital radius of the solar nebula as one light year. The value of $R_{1}$ as obtained by making use of the inner planets comes out to be about 0.1 light year. The discrepancy in the two value of the orbital radius of the solar nebula, as obtained from the data of the outer and the inner groups of the planets respectively, may be on account of the uncertainties in the parameters of the latter. The maximum uncertainty seems to be in 
the value of $K$. As the composition of the terrestrial planets differs appreciably from that of the Sun, it seems that the present mass of these planets are much difterent from the mass of their original gaseous rings. A multiplication factor as much high as 400 is obtained (21) if these planets be considered to posses originally the same composition as that of the Sun. Therefore the value one light year of the orbital radius of the solar nebula, as obtained from the outer group) of the planets, may only be considered.

The solar nebula and its brother star were revolving round their parent star (of mass equal to $\left.10^{6}\right)$ ) at the time of the formation of their planetary system. Since such a heavy star could not remain stable for long time, the parent star must have been subdivided into smaller bodies after turning into a nova or supernova. At present therefore such a heavy star is not to be found in the neighbour hood of the Sun. However, the brother star of the Sun may still be existing at approximately the same distance which according to the present study is of the order of a light year. This distance is in agreement with the observed distance of some closest stars to the Sull.

\section{MISctission.}

The theory in its present form using some assumptions and approximations can be expected to give results only within a certain limited degree of accuracy. However the consistency of the results and ideas is striking. The consistencies are enumerated as follows:

(a) The angle $\theta$ used in Eq. [21] has been argued to depend upon the total number of the planets formed in any sequence and its value was predicted to be nearly $8.5^{\circ}$ for a.sequence of live bodies and $17^{\circ}$ for a sequence of four bodies. After adjusting the parameters it was found that these values or values close to them could only satisfy Eq. [21]. These values of $\theta$ consistently satisfy not only the two sequences of the planets but also a number of sequences of the satellites.

(b) The prediction that $\theta_{0}$ should be zero for all such sequences of bodies, beyond which already there exist some bodies, proved to be consistently correct for all the planetary and satellite systems. All the satellite sequences studied here are similar to the inner group of the planets because for all such sequences some bodies of higher orbits exist. For all these systems $\theta_{0}$ is actually found to be zero. 
For the outer group of the planets beyond which no more planets are known to exist, the value of $\theta_{0}$ is finite as expected.

(c) The constant $B$ solely depends upon the ratio of the orbital radii of the Sun and its brother star. Taking $R_{2} / R_{1}=2.5$, the tentative value of $B$ was obtained and used for the planetary system. This value was found to obey the outer as well as the inner group of the planets. It is exactly what is expected on the basis of the proposed theory because the same brother star of the Sun has produced both the groups of the planets in its two successive approaches towards the Sun. A wide gap observed between the outer and the inner group of the planets corresponds to that phase of the orbital motion of the brother star in which it recedes from the Sun. A large number of asteroids observed in this region may be small bodies condensed from the remnant gases which were left during the contraction of the solar nebula between the outer and inner group of the planets. The value of the constant $B$, as obtained by substituting the observed value of $R_{2} / R_{1}$ in $[13 \mathrm{~b}]$, fits well in all the satellite system too. Moreover the value of $B$ for the satellite systems of Jupiter and Saturn are the same as is expected. Both these planets have produced the satellite systems of each other and therefore the value of $B=\frac{2 R_{1} R_{2}}{R_{1}{ }^{2}+R_{2}{ }^{2}}$ should be the same for the two.

(d) The maximum support to the proposed theory comes from the consistency with which the empirical values of the constant $A$ agree with the values obtained from the expression [13a]. Various quantities occurring in the expression for $A$ differ widely in their order of magnitude but on substitution, these quantities could give the correct order of magnitude of $A$ as obtained by adjusting the parameters of Eq. [21]. More accuracy cannot be expected in view of the use of approximate values of some of the quantities e.g. $\eta_{0}, K$, $n$ and $n^{\prime}$.

It would be worthwhile to discuss the various factors which limit the degree of accuracy of our results:

(1) The density distribution of the form $\varrho=\frac{K}{r^{n}}$ is expected to be true only approximately. The value of the index $n$ is not known in any case from rigorous considerations. Moreover the determination of the constant $K$ needs the external and internal radii and the mass 
of the gaseous ring. These can be known only approximately under some simplified assumptions with the help of the mass and the orbital radius of the planet formed out of the gaseous ring. The simplifying assumptions are that the mass of the gaseous ring is equal to the mass of the planet and the gaseous ring is condensed at the centre of gravity of its radial mass distribution.

(2) The temperatures of the different layers of the nebular disc at the respective times of their splitting are assumed to be of the form $\Theta \propto \frac{1}{r^{n}}$. The temperature is, no doubt, expected to increase with the depth of the layers but the index $n^{\prime}$ is not known precisely.

(3) An approximate composition of the gases in the nebula has been considered.

(4) Besides the above approximations, the validity of Eq. [21] depends upon the assumption regarding the timings at which different gaseous rings were formed. Though some justification has been given for the assumption that the time of the formation of any gaseous ring will be double of the time of the formation of its preceding ring, still it needs further discussion.

\section{Conclusion.}

The present theory provides the following features regarding the origin and evolution of the solar system:

1. At the time of its formation the Sun was not only revolving round the centre of our galaxy but atleast about one more intermediatory body along with few fellow stars in a manner as planets revolve round the Sun.

2. In the earlier stages of their formation both the solar nebula and its brother star (also a nebula) contracted gravitationally under the tidal force of each other till the outermost layer in the boundary of each is nearing Keplerian velocity. As this stage the viscous force became appreciable and did not allow any boundary layer to attain exact Keplerian velocity.

3. During that part of the orbital motion of the Sun and its brother star when they approached each other, sufficient energy was 
received by these boundary layers to overcome the viscous force and this enables them to attain exact Keplerian velocity. Consequently a boundary layer split from the main body in the form of a rotating gaseous ring.

4. A series of such rings were split at definite intervals of time. The time taken by any gaseous ring for its splitting was double that of its preceding ring. These gaseous rings later on condensed by some unknown process in their own orbits to give rise to the planets.

5. The law of distance obtained on the basis of the above contention is not only satisfied by the planets but also by the satellites showing thereby that the theory is also applicable to the formation of the satellites.

6. The same law of distance, when applied to the formation of Sun and its brother star, gives the position of the parent star of the Sun at the time of the formation of the Sun. The distance of the parent star from the San was of the order of a light year.

\section{ACKNOWLEDGEMLNT.}

The author is grateful to Dr. P. K. SHarma, Physics Department, Allahabad University - Nlahabad - for helpful discussion and guidance throughout the present investigation.

\section{REFERENCES}

(1) Alfvén II., Stockholms Obs. Ann. 14 (1942).

(2) Alrván II., Stockholms Obs. Ann. 14 (1943).

(3) Alrvin II., Stockholms Obs. Ann. 14 (1946).

(4) Alfves II., On the origin of the Solar System, Oxford "Univ. Press." New York (1954).

(5) Alyves H., lcarus, 3, 57 (1964).

(') Alrven II., On the Origin of the Solar System. Harold Jeffrey's lectures at the Royal Astronomical Society, London. Monthly Notices, 87 (1967a).

(7) Alfvén II., Accretion of Planets. Preprint. Technical Report Space Sciencie Laboratory; Berkeley, Series No 8, Issue No 105, U.C., p. 15 (1956b).

(8) Alfvén H., learus 7, 387 (1967e). 
$\left({ }^{9}\right)$ Arrilenius S., Das Werden der Welten, Leipzig (1913).

$\left({ }^{10}\right)$ Berlaage Jr. H. P., Ergänzungshand Gerlands Beitr. Geophis. 17 (1927).

(11) BerLage Jr. H. P., IJet ontstaan en vergaan der werelden, Amsterdam (1930a).

(12) Berlafie Jr. H. P., Proc. Koninkl. Ned. Akad. Wetenschap. (Amsterdaun) 33, 614, $719(1930 \mathrm{~b})$.

(13) Bickerton A. W., 'Trans. New Zealand Inst. 11, 125 (1878).

$\left({ }^{14}\right)$ Birkeland K., Compt. rend. acad. Sci. 155, 892 (1912).

$\left({ }^{15}\right)$ Buffon G. L. L., De la formation des planètes, Paris (1745).

${ }^{(16)}$ Chamberdain 'T. C., The Origin of the Earth, Chicago (1927).

(17) Descartes R., Principia Philosophiae. Amsterdam (1644).

(18) Dorosikevitci A. G., Zeldovic Ya. B., Nivikov I. D., Astron. Zh., (U.S.S.R.) 44. 295 (1967).

(19) Giulı 'T., Icarus, 8, 301 (1968).

$\left({ }^{20}\right)$ IIoyle F., Monthly Notices Roy. Astron. Soc. 106. 406 (1946).

${ }^{(1)}$ Hoy t.e F., Proceedings of the Conjerence on the Origin of the Solar Sys. em. (Ed. by Jastrow R. and Cameron A. G. W.) Academic Press, New York p. 63, (1962).

$\left({ }^{22}\right)$ Jeans J. H., Astronomy and Cosmogony, Cambrilge Univ. Press. New York (1928).

$\left.{ }^{(23}\right)$ Jefrreys H., The Earth, Cambridge Liniv. Press, New York (1924).

(24) Jeffreys H., Monthly Notices Roy. Astron. Soc. 89, 636 (1929).

$\left.{ }^{25}\right)$ Kant I., Allgemeine Natursgeschichte und Theorie des Irimmels. (1755).

$\left({ }^{26}\right)$ Kuiper G. P., Proc. Natl. Acal. Sc. U..S. 37, 1 (1951).

(27) LAPLACE P. S., Exposition du système $d u$ Monde, Paris (1796).

${ }^{(28)}$ Lytrteton R. A., Monthly Notices Roy. Astron. Soc. 98, 536 (1938a).

(29) Lyttideton R. A., Monthly Notices Roy. Astron. Soc. 98, 646 (1938b).

$\left({ }^{30}\right)$ Marcus A. II., Icarus, 7, 283 (1967).

(31) Mitra V., Silarma P. K., Memorie della Soc. Astronom. Italiana, 39. 459 (1968).

${ }^{(32)}$ Moulton F. R., Astrophys. J. 22, 165 (1905).

${ }^{(33)}$ Russel. I. H. N., The Solar System and its Origin. Mcmillan, New York $(1935)$.

(34) Scmumt O., A theory of the Origin of the Earth; Four Lectures. Lawrence \& Wishart, London (1959).

${ }^{35}$ ) Spitzer I., Astrophys. J. 90.675 (1939).

$\left({ }^{36}\right)$ Ter Haar I), Proc. Roy. Soc. Edinburgh 64, 1 (1953).

$\left({ }^{37}\right)$ Von Weizsäcker C. F., Z. Astrophys. 22, 319 (1944).

${ }^{(38)}$ WiIPPle F. L., Harvard Obs. Monographs, No 7 (1948). 